\title{
Biochemical and Genetic Characterization of Streptomyces rimosus Mutants Impaired in Oxytetracycline Biosynthesis
}

\author{
By P. M. RHODES, ${ }^{*}$ N. WINSKILL, E. J. FRIEND AND M. WARREN \\ Pfizer Central Research, Sandwich, Kent CT13 9NJ
}

(Received 22 September 1980)

\begin{abstract}
Antibiotic non-producing mutants were isolated from an oxytetracycline (OTC) producing strain of Streptomyces rimosus. Cosynthesis tests and feeding known intermediates of the OTC pathway allowed the classification of the mutants into several groups. The biosynthetic lesion was determined for several of the mutants. Some of the mutants deficient in the reduction of 5a,11a-dehydrooxytetracycline, the final step in the pathway, were unable to synthesize a cofactor (CSF1) essential for this reaction. Mutations apparently in genes for enzymes of the main OTC pathway were found for three of a possible four steps between 4-aminodedimethylaminoanhydrotetracycline (4-amino-ATC) and OTC. Mutations affecting three other steps of a possible five before 4-amino-ATC were found, but unambiguous identification of these was not possible. The genes for OTC production, including those for CSF1 synthesis, were located in two diametrically opposite clusters on the $S$. rimosus chromosomal linkage map. No evidence for plasmid-borne genes was obtained.
\end{abstract}

\section{INTRODUCTION}

Despite the extensive use of blocked mutants for the elucidation of the terminal steps of the biosynthesis of the antibiotic chlortetracycline by Streptomyces aureofaciens (McCormick, 1966, 1968), the lack of efficient methods for genetic analysis has prevented the study of gene-enzyme relationships in this pathway (Blumauerova et al., 1972). In contrast, a well developed genetic system exists in Streptomyces rimosus which produces the related compound oxytetracycline (OTC) (Friend \& Hopwood, 1971; Alačević et al., 1973). Although mutants impaired in OTC production have been reported (Delic et al., 1969; Alikhanian et al., 1961) and preliminary gene mapping has been described (Boronin \& Mindlin, 1971; Alačević, 1976), the biochemical nature of the lesions has not been studied.

In this paper we describe the isolation of OTC non-producing mutants, the investigation of the biosynthetic blocks, and the position of the genes involved on the $S$. rimosus linkage map. This is the first combined study of the biochemistry and genetics of OTC production.

\section{METHODS}

Strains. All the strains of Streptomyces rimosus (Table 1) were derived from a prototrophic strain used commercially for production of OTC (Friend \& Hopwood, 1971). Streptomyces aureofaciens ATCC 13190 (Miller et al., 1960) was used as a source of CSF1. A strain of Bacillus subtilis from the Pfizer collection was used in bioassay procedures for antibiotic detection.

Media. Complete medium (CM) and minimal medium (MM) were as described by Friend et al. (1978). Liquid complete medium (LM) contained, per litre: D-glucose, $10 \mathrm{~g}$; yeast extract, $5 \mathrm{~g}$; lactalbumin hydrolysate, $15 \mathrm{~g}$; sucrose, $2.8 \mathrm{~g}$; calcium carbonate, $1 \mathrm{~g}$.

Mutagenesis. Three mutagens were used for the production of OTC-negative mutants: $N$-methyl- $N^{\prime}$ nitro- $N$-nitrosoguanidine (Delić et al., 1970); ultraviolet light of wavelength $254 \mathrm{~nm}$; ultraviolet light of wavelength $365 \mathrm{~nm}$ after sensitization with 8-methoxypsoralen (Townsend et al., 1971). 


\section{Table 1. Streptomyces rimosus strains}

\begin{tabular}{ccl}
$\begin{array}{c}\text { Strain } \\
\text { no. }\end{array}$ & $\begin{array}{c}\text { OTC mutation } \\
\text { no. }\end{array}$ & \multicolumn{1}{c}{ Genotype } \\
R260 & OTC producing & adeA2 hisA1 \\
R261 & OTC producing & argA1 lysB2 \\
R828 & OTC producing & proA3 uraA8 \\
R425 & OTC producing & proA3 lysB2 metB2 \\
K1378 & 4 & argA1 lysB2 otcD4 \\
R902 & 4 & ribB1 cysD3 otcD4 \\
K1581 & 19 & adeA2 hisA1 csfA19 \\
K1573 & 20 & adeA2 hisA1 otcX20 \\
K1591 & 25 & hisA1 otcA25 \\
K1626 & 56 & adeA2 hisA1 csfB56 \\
K1645 & 75 & adeA2 hisA1 otcC75 \\
K1660 & 90 & adeA2 hisA1 otcY90 \\
K1772 & 151 & argA1 lysB2 otcZ151 \\
K1727 & 155 & csfC155
\end{tabular}

Isolation of OTC non-producing mutants. Mutagen-treated spore suspensions were spread on the surface of CM plates and incubated at $28{ }^{\circ} \mathrm{C}$ until colonies were just visible to the naked eye (about $2 \mathrm{~d}$ ). Colonies, each on a cylinder of agar, were removed from the plates using a sterile cork borer of $4 \mathrm{~mm}$ diameter. Incubation was continued in a humid environment for a further $5 \mathrm{~d}$. The plugs were then placed on the surface of antibiotic assay agar (Difco) seeded with Bacillus subtilis. After overnight incubation at $37^{\circ} \mathrm{C}$ parental colonies produced an inhibition zone of diameter $25 \mathrm{~mm}$.

Cosynthesis. Three different procedures were used.

(1) Agar mixed culture test. Spore suspensions $\left(10^{6}-10^{7}\right.$ spores $\left.\mathrm{ml}^{-1}\right)$ of two strains were mixed on the surface of $4 \mathrm{~mm}$ diameter cylinders of $\mathrm{CM}$ and incubated at $28^{\circ} \mathrm{C}$ in a humid chamber for $5 \mathrm{~d}$. Antibiotic was assayed by placing the cylinder on antibiotic assay agar (Difco) seeded with B. subtilis and incubating overnight at $37^{\circ} \mathrm{C}$. A halo of inhibited growth indicated cosynthesis.

(2) Agar strip test. This was an adaptation of the procedure of Delic et al. (1969). Spore suspensions of two strains were separately spread on each half of a Petri dish containing CM, with a $3 \mathrm{~mm}$ wide uninoculated strip between them. After incubation at $28{ }^{\circ} \mathrm{C}$ for $5 \mathrm{~d} \mathrm{a} 10 \mathrm{~mm}$ wide strip of agar was cut from the plate, perpendicularly to the uninoculated strip. The agar strip was placed on $B$. subtilis agar and antibiotic production was indicated by a halo of inhibited growth around one of the strains on the strip. That strain was assumed to have produced OTC by cosynthesis.

(3) Liquid culture test. Spore suspensions of two strains were inoculated into $45 \mathrm{ml} \mathrm{LM}$ in a $300 \mathrm{ml}$ Erlenmeyer flask. The concentration of each strain in the medium was $10^{6}$ spores $\mathrm{ml}^{-1}$. Flasks were shaken at $220 \mathrm{rev}$. min ${ }^{-1}$ on a rotary shaker with a $4 \mathrm{~cm}$ stroke, at $28^{\circ} \mathrm{C}$. After incubation for $4 \mathrm{~d}$ the culture was acidified with $1 \mathrm{M}-\mathrm{HCl}$ to pH 2 and filtered through Whatman no. 1 paper. The filtrate was assayed for OTC by high performance liquid chromatography as described below.

Genetic mapping of OTC-negative mutations. The location of OTC-negative mutations relative to previously mapped auxotrophic markers was determined by haploid recombinant analysis (Hopwood, 1967b). Crosses between an OTC non-producing mutant and an OTC producing parent carrying complementary auxotrophic markers were plated on several different selective media to recover recombinants. Samples of the recombinants were purified by streaking twice on to the same selective medium to exclude contamination with parental spores. Each purified recombinant colony was inoculated as a patch on a master plate and on a plug of CM. Master plates were incubated for 3-4 d and then replicated to diagnostic media; agar plugs were incubated for $5 \mathrm{~d}$ and assayed for antibiotic activity against $B$. subtilis as described above. Each recombinant was classified for inheritance of auxotrophic markers and production of antibiotic. The size of the inhibitory zones produced by recombinants depended on the particular combination of markers inherited. However, scoring recombinants on the criterion of no inhibition versus some inhibition was unambiguous. Consideration of allele frequencies amongst recombinants gave two possible map locations for each OTC mutation (in either arc between the selected markers). The 'correct' alternative was that which minimized the crossovers required to produce the observed progeny of recombinants.

Chromatography. High performance liquid chromatography (HPLC) of OTC-containing extracts was carried out using a Waters Associates model ALC 200 chromatograph under the following conditions: column, Waters $\mu$ Bondapak $\mathrm{C}_{18}(250 \mathrm{~mm} \times 4 \mathrm{~mm}$ i.d.); mobile phase, acetonitrile/water (1:4) containing $0.1 \mathrm{M}$-perchloric acid; flow rate, $2.5 \mathrm{ml} \mathrm{min}^{-1}$; detector, absorbance at $254 \mathrm{~nm}$. 
Chemicals. Oxytetracycline, anhydrotetracycline (ATC), 4-aminodedimethylaminoanhydrotetracycline (4amino-ATC), and 7-chloro-5a(11a)-dehydrotetracycline (7-chloro-DHTC) were Pfizer laboratory reference compounds.

\section{RESULTS}

\section{Isolation of OTC-negative mutants}

The choice of parental strains for the isolation of antibiotic-negative mutants was made to facilitate subsequent genetic analysis. A pair of strains (R260 and R261, see Table 1) carrying complementary auxotrophic mutations of known positions on the circular chromosome (Friend \& Hopwood, 1971) was selected; none of the auxotrophies prevented the production of OTC under the test conditions.

Colonies giving significantly smaller antibiotic inhibition zones than the parent strain were found at an average frequency of $0.5 \%$ after mutagenesis. These were isolated, purified and retested. After the elimination of leaky mutants and those sensitive to growth inhibition by tetracycline antibiotics, 57 mutants were selected out of about 40000 colonies examined $(0.14 \%)$. None of the selected mutants were grossly abnormal in colony size or morphology.

The OTC producing ability of the 57 mutants in liquid culture was tested by HPLC. An undetectable amount (less than $1 \mu \mathrm{g} \mathrm{ml}^{-1}$ ) of OTC was produced by 32 of the mutants. The remaining mutants produced $10-20 \mu \mathrm{g} \mathrm{ml}^{-1}$ under the test conditions, compared with the parent strains which produced $1000-2000 \mu \mathrm{g} \mathrm{ml}^{-1}$.

\section{Cosynthesis between OTC-negative mutants}

The classification of the mutants impaired in OTC biosynthesis was first attempted using the principle of cosynthesis (McCormick et al., 1960). This process is the counterpart in non-essential metabolism of the phenomenon of syntrophism between auxotrophic mutants. When two OTC non-producing mutants of $S$. rimosus are grown together in close association and produce OTC, they are said to show cosynthesis. The underlying mechanism of this co-operative biosynthesis could theoretically result from (i) genetic repair via recombination, (ii) transfer of an enzyme or coenzyme from one culture to the other, or (iii) transfer of a biosynthetic precursor of OTC from one strain to the other (McCormick, 1966). If the final explanation is correct, the method affords an opportunity of deciding if two mutants are blocked at the same or different steps in the OTC pathway; cosynthesis then indicates blocks at different points, while lack of cosynthesis may indicate blockage at the same point. Alternatively, a failure to observe cosynthesis could result from the failure of a mutant to excrete the intermediate preceding the block, perhaps because of impermeability of the cell to the compound, or to chemical instability of the compound. Three different experimental procedures to demonstrate cosynthesis between OTC non-producing mutants were used in the present study, and the results obtained depended on the procedure used.

In the first procedure, two OTC non-producing mutants were grown together on the surface of an agar plug. Several hundred such tests were carried out, involving all 57 mutants, and the results showed that at least nine different types of mutant were present. Representative mutants of each type (otc-4, $-19,-20,-25,-56,-75,-90,-151$ and -155 ) were selected for more detailed examination. These cosynthesized in all possible pair combinations, confirming that nine different OTC non-producing mutations were present. The second procedure, the agar strip method, was designed to elucidate the sequence of the biosynthetic blocks. The nine representative mutants were tested in all possible pair combinations. The results were similar to those obtained previously in $S$. rimosus by Delic et al. (1969). The mutants could be divided into two classes on the basis of the cosynthetic patterns. Class 1 mutants (otc-19, -56 and -155 ) rarely demonstrated cosynthesis when grown with each other; positive results were equivocal and non-reproducible. Class 2 mutants (otc-4, -20, $-25,-75$, -90 and -151 ) also showed only infrequent and non-reproducible positive results. In contrast, 
Table $2 .{ }^{14} \mathrm{C}$ content of OTC produced by cosynthesis

\begin{tabular}{|c|c|c|c|}
\hline $\begin{array}{c}\text { Mutant } \\
\text { combinations }\end{array}$ & $\begin{array}{l}\text { OTC produced } \\
\left(\mu \mathrm{g} \mathrm{ml}^{-1}\right)\end{array}$ & $\begin{array}{l}\text { Total activity } \\
\text { in OTC }(\mu \mathrm{Ci})\end{array}$ & $\begin{array}{c}\text { Specific activity } \\
\left.\text { of OTC }(\mu \mathrm{Ci} \mathrm{mg})^{-1}\right)\end{array}$ \\
\hline $\begin{array}{l}\text { A) } o t c-4^{*}+o t c-151 \\
\text { 3) } o t c-4+o t c-151^{*}\end{array}$ & $\begin{array}{l}220 \\
150\end{array}$ & $\begin{array}{l}1.23 \times 10^{-2} \\
3.10 \times 10^{-3}\end{array}$ & $\begin{array}{l}1.60 \times 10^{-3} \\
5.74 \times 10^{-4}\end{array}$ \\
\hline atio $(\mathrm{A} / \mathrm{B})$ & & 3.97 & $2 \cdot 81$ \\
\hline
\end{tabular}

* Indicates the ${ }^{14} \mathrm{C}$-labelled partner.

No OTC was produced in controls with mutant combinations otc- $4^{*}+$ otc-4 and otc-151* + otc-151.

when a class 1 mutant was grown with a class 2 mutant, the agar strip test always gave cosynthesis of OTC, the class 1 strain always acting as the converter and the class 2 strain always as the secretor. Thus, we were not able to use the agar strip procedure to determine a simple consistent sequence for the blocks in OTC biosynthesis, and the results obtained with this procedure disagreed with those obtained in mixed culture. It seemed pcssible that the failure to observe cosynthesis in the agar strip method in some cases must be due to the failure of a compound to cross the $2-3 \mathrm{~mm}$ gap between the two strains when grown separately in this way.

The third method consisted of growing pairs of mutants together in liquid medium in shaken flasks. The observations on mutants grown together in mixed culture on agar plugs were confirmed, and it was also confirmed that the antibiotically active product was OTC.

\section{Transfer of ${ }^{14} \mathrm{C}$-labelled OTC precursors}

In an attempt to provide direct evidence that OTC precursor exchange was taking place in cosynthesizing mixed cultures, and to decide on the sequence of blocks in the OTC pathway, a radiotracer method of analysis was devised. Radiolabelled acetate, a precursor of OTC, was added to the growth medium of one strain in order to label the intracellular pool of OTC precursors. The mycelium was harvested, washed free of excess acetate by centrifugation and inoculated into fresh medium which contained unlabelled mycelium of the other strain. After further incubation, the OTC produced was recovered from the medium and its radiochemical content was determined. If cosynthesis was occurring by transfer of a biosynthetic intermediate, then labelled OTC should be formed when the secretor strain was labelled, but not when the converter strain had been labelled.

The results shown in Table 2 demonstrate this phenomenon in a pair of class 2 mutants. The specific radioactivity of the OTC produced when the labelled strain was otc-4 was 2.8 times greater than when otc-151 was labelled, showing that otc-4 was blocked later in the OTC pathway. The $\left[{ }^{14} \mathrm{C}\right] \mathrm{OTC}$ formed when otc-151 was labelled could have arisen from secretion of a labelled early intermediate by otc-151, partial conversion to a later intermediate by otc- 4 and then terminal reaction(s) being completed by otc-151. Other pairs of mutants tested in this type of experiment failed to cosynthesize, perhaps because of damage to the mycelium during the washing procedure. Improvements in technique may be required to extend the usefulness of this new method.

\section{Response of OTC-negative mutants to cosynthetic factor 1}

Miller et al. (1960) showed that certain mutants of $S$. aureofaciens were unable to produce chlortetracycline because they lacked a flavin-like cofactor, which they termed cosynthetic factor 1 (CSF 1), required in catalytic amounts for the final step in the biosynthetic pathway. Since an identical step occurs in OTC biosynthesis (Miller et al., 1965), we determined if any of our $S$. rimosus mutants also lacked this cofactor. 


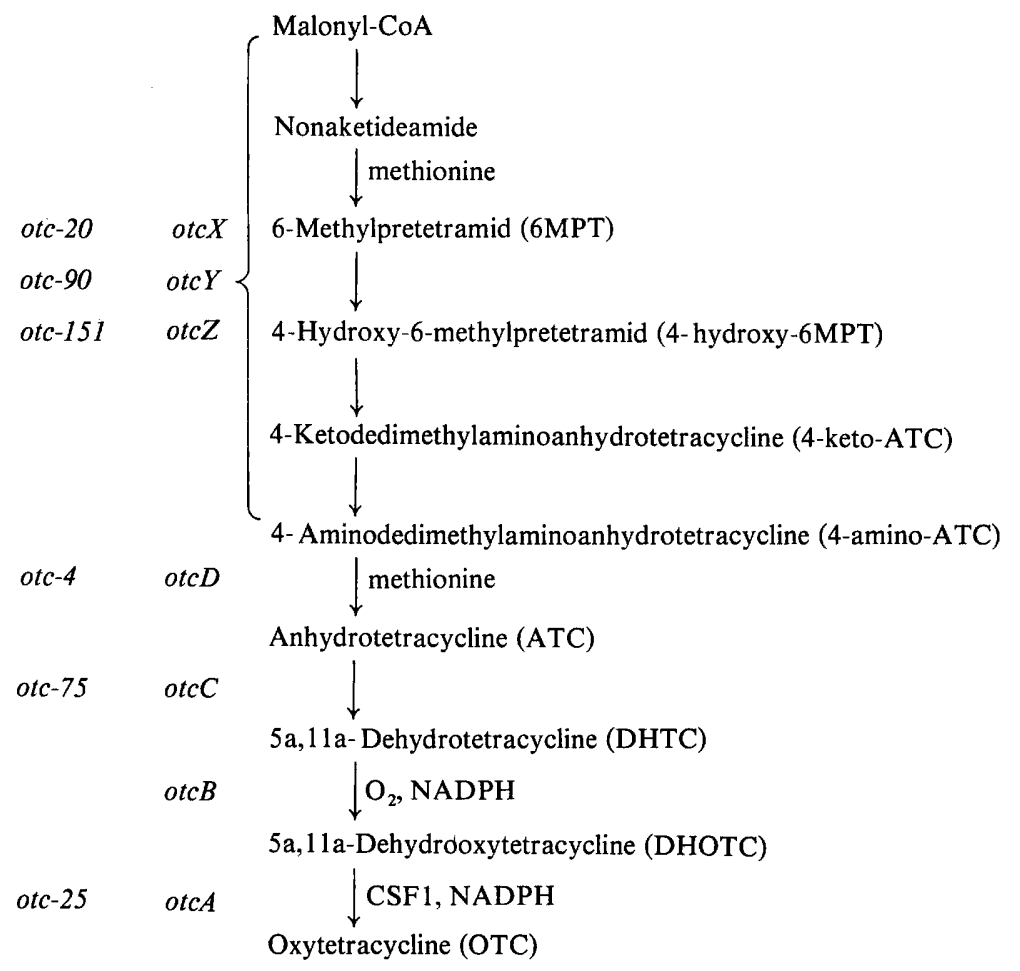

Fig. 1. Biosynthesis of oxytetracycline, indicating the steps at which various OTC-negative mutants are blocked.

Culture filtrate of the CSF1 producing strain S. aureofaciens ATCC 13190 (Miller et al., 1960) was supplied to each OTC mutant. All three class 1 mutants (otc-19, -56, -155), but no class 2 mutant, responded by producing OTC. The requirement for a catalytic quantity of active substance was shown by demonstrating the same effect with a 1000 -fold diluted culture filtrate of S. aureofaciens ATCC 13190.

Class 1 mutants failed to stimulate the production of chlortetracycline by the CSF 1 non-producing mutant $S$. aureofaciens ATCC 12748 (McCormick et al., 1960), and so they evidently did not excrete CSF1. Conversely, all six class 2 mutants (otc-4, -20, -25, -75, -90 and -151 ) produced CSF 1 as indicated by this test.

All these results are consistent with the hypothesis that the class 1 mutants are each blocked in the CSF1 biosynthetic pathway, at various points. CSF1 is known to be chemically stable and can be transferred from cell to cell by diffusion. Some CSF1 non-producing mutants, e.g. otc-155 and ATCC 12748, were found to be auxotrophic for riboflavin suggesting the existence of one or more common biosynthetic steps. The three CSF1 non-producing mutants otc-19, -56 and -155 were assigned to genes csfA19, csfB56 and $c s f C 155$, respectively.

\section{Conversion of precursors to OTC}

Having established that the class 1 mutants were deficient in CSF1, attention was turned to the biochemical nature of the lesions in OTC biosynthesis in each representative class 2 mutant. Cosynthesis results in mixed cultures had indicated at least six different groups of such mutants.

OTC precursors were supplied to liquid cultures of all class 2 mutants, and their conversion, or lack of conversion, to OTC was determined by HPLC and by bioassay. When 
Table 3. Conversion of precursors to OTC (or 7-chloro-OTC) by class 2 mutants

\begin{tabular}{lccc}
\multirow{4}{*}{ Mutant } & \multicolumn{3}{c}{$\begin{array}{c}\text { Percentage conversion of precursor to } \\
\text { 7-chloro-OTC or OTC }\end{array}$} \\
\cline { 2 - 4 } otc-20 & 7-Chloro-DHTC* & ATC & 4-Amino-ATC \\
otc-90 & + & 30 & 44 \\
otc-151 & + & 49 & 36 \\
otc-4 & + & 86 & 4 \\
otc-75 & + & 105 & 0 \\
otc-25 & + & 0 & 0 \\
& - & 0 & 0
\end{tabular}

* Production of 7-chloro-OTC was estimated by bioassay only: + , production; - , no detectable production.

the supplied compound was converted to OTC, the mutant was assumed to be blocked at a step in the OTC biosynthetic sequence prior to that intermediate. An outline of the sequence, incorporating the results of Miller et al. (1965), is given in Fig. 1. The earlier steps in OTC biosynthesis were assumed to be analogous to the equivalent steps in chlortetracycline synthesis, although this has never been formally demonstrated. Available intermediates were fed to the six representative class 2 mutants (Table 3 ).

The final intermediate before OTC, DHOTC, has apparently never been isolated and therefore was not available for test. The penultimate intermediate, DHTC, is unstable and therefore also was not available for testing. The 7-chloro derivative, however, is stable and is converted by $S$. rimosus into 7-chloro-OTC (Martin et al., 1966). The presence of 7-chloro-OTC in fermentation broths was established by the following criteria: antibiotic potency, ultraviolet spectrum, and HPLC behaviour. Mutants otc-4, $-20,-75,-90$ and -151 were able to carry out this conversion. Mutant otc-25, which could not carry out this conversion, might have been blocked either between OTC and DHOTC or between DHTC and DHOTC (Fig. 1), or might have been blocked at more than one step, perhaps through a regulatory defect causing repression of the whole OTC pathway. If the mutant were defective in the conversion of DHTC to DHOTC, then it should produce an antibiotically active substance, tetracycline; this was not the case. The third possible reason for OTC non-production by otc- 25 is eliminated because of its ability to cosynthesize with mutants blocked earlier in the OTC pathway (such as otc-75, see below). This shows that otc-25 is capable of generating an OTC precursor such as DHTC or DHOTC (Fig. 1) and cannot therefore be deficient in any step before DHTC. Thus, otc- 25 can only be blocked between DHOTC and OTC; it was assigned to gene otcA.

ATC was converted to OTC by all the class 2 mutants tested except otc- 25 and -75 . Mutant otc-75 could convert 7-chloro-DHTC to 7-chloro-OTC and must therefore be blocked between ATC and DHTC. Mutant otc- 75 was assigned to gene otcC.

4-Amino-ATC was converted to OTC by all the class 2 mutants tested except otc-25, -75 and -4 . Since otc-4 could convert ATC and 7-chloro-DHTC to OTC and 7-chloro-OTC, respectively, it must be blocked between 4 -amino-ATC and ATC. Mutant otc-4 was assigned to gene otcD.

None of the mutants apparently blocked before 4-amino-ATC was able to convert the earlier precursors 4-hydroxy-6MPT or 6MPT to OTC. Since a negative result might have been due to deficiencies in experimental method, no definite conclusion could be drawn. However, cosynthesis data showed that three different blocks were present, and so the mutants otc-20, -90 and -151 were assigned to genes otcX, otc $Y$ and otcZ, respectively. Further work is required to clarify the nature of the deficiencies in these mutants. 


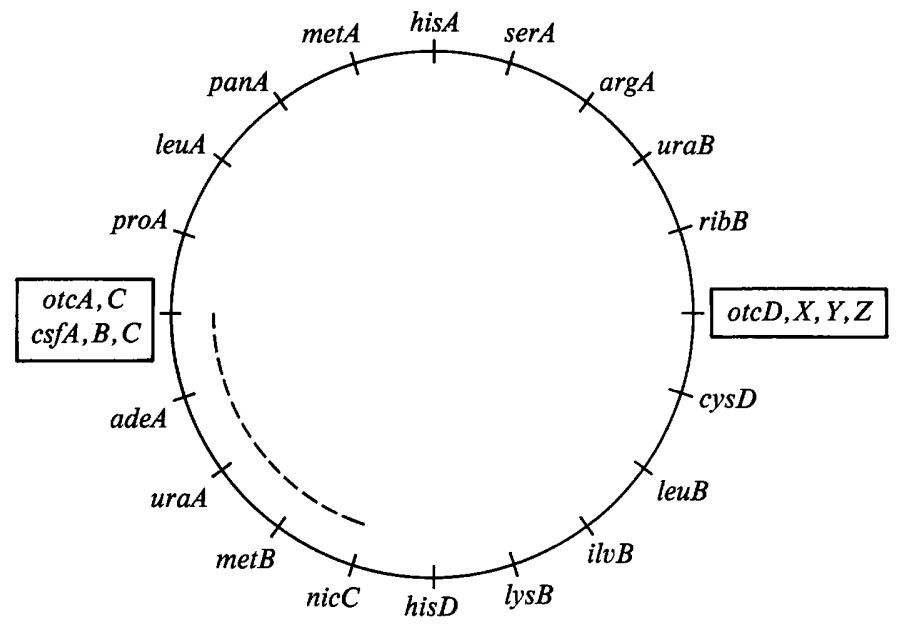

Fig. 2. The location of OTC-negative mutations on the genetic map of $S$. rimosus. The loci are arbitrarily spaced at equal intervals. The dashed line indicates the region of the otc genes reported by Pigac et al. (1974).

Table 4. Location of otcD4 in a cross of $R 902$ (proA3 ${ }^{+}$ribB1 uraA $^{+}$cysD3 otcD4) with

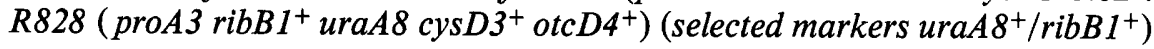
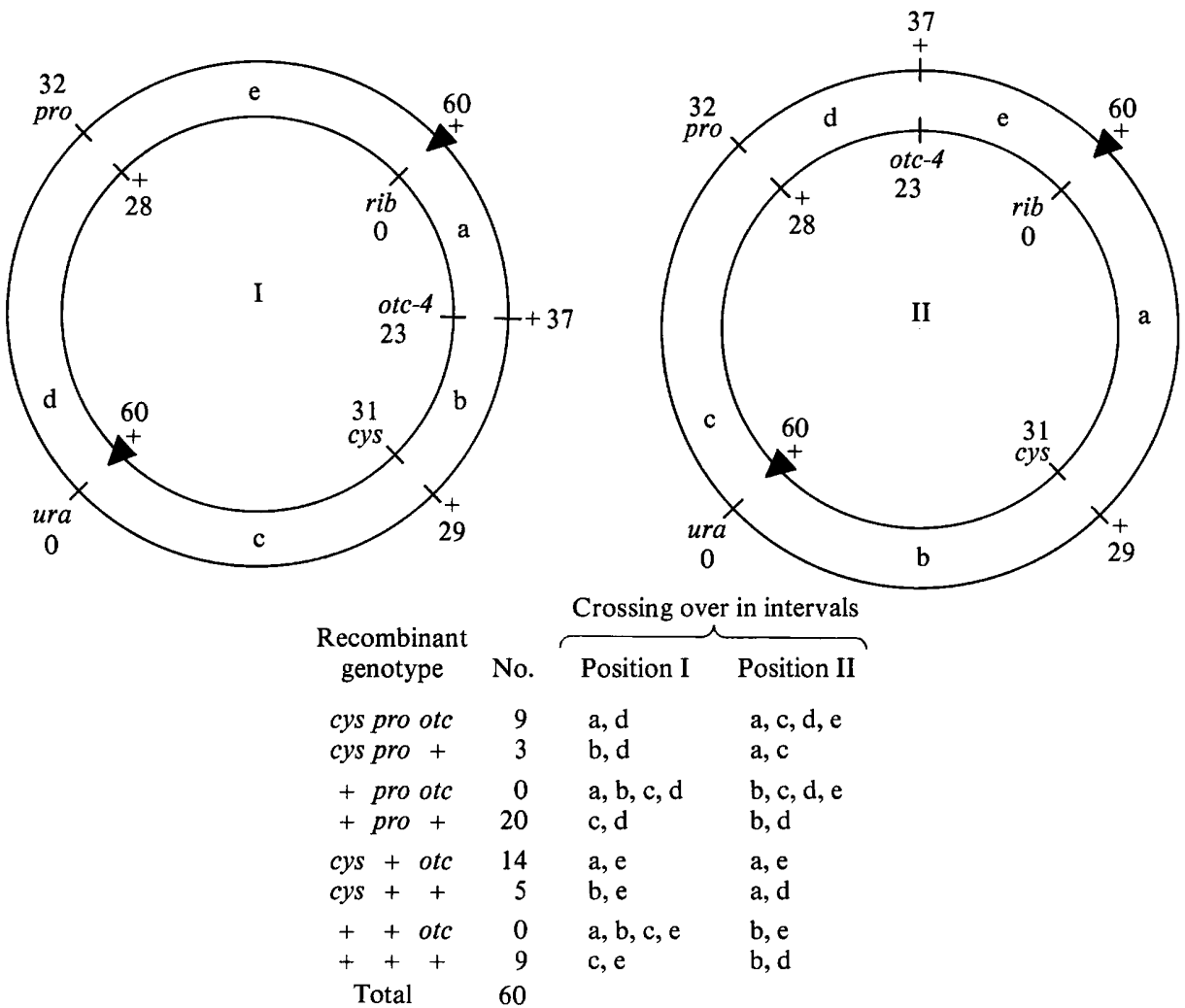

Crossing over in intervals

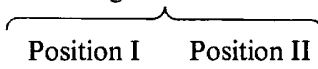

$\begin{array}{ll}\text { a,d } & \text { a,c,d,e } \\ \text { b, d } & \text { a,c } \\ \text { a, b, c, d } & \text { b, c, d, e } \\ \text { c, d } & \text { b,d } \\ \text { a, e } & \text { a,e } \\ \text { b, e } & \text { a,d } \\ \text { a, b, c, e } & \text { b, e } \\ \text { c, e } & \text { b, d }\end{array}$

The two map locations for otc-4 indicated (I and II) are consistent with the observed allele ratio otc-4/otc-4 ${ }^{+}$ Position $I$ is chosen because this location requires only two crossovers to generate all recombinant classes, including the nine cys pro otc recombinants. This recombinant class would require four crossovers if position II were correct. 
Table 5. Location of otcC75 in a cross of $K 1645$ (proA3 $3^{+}$adeA 2 lys B2 ${ }^{+}$metB2 $2^{+}$hisA 1 otcC75) with $\mathrm{R} 425$ ( $\mathrm{proA} 3$ adeA2 $2^{+}$lysB2 metB2 hisA $1^{+}$otc $\mathrm{C75}^{+}$) (selected markers ade $A 2^{+} /$lys $B 2^{+}$)
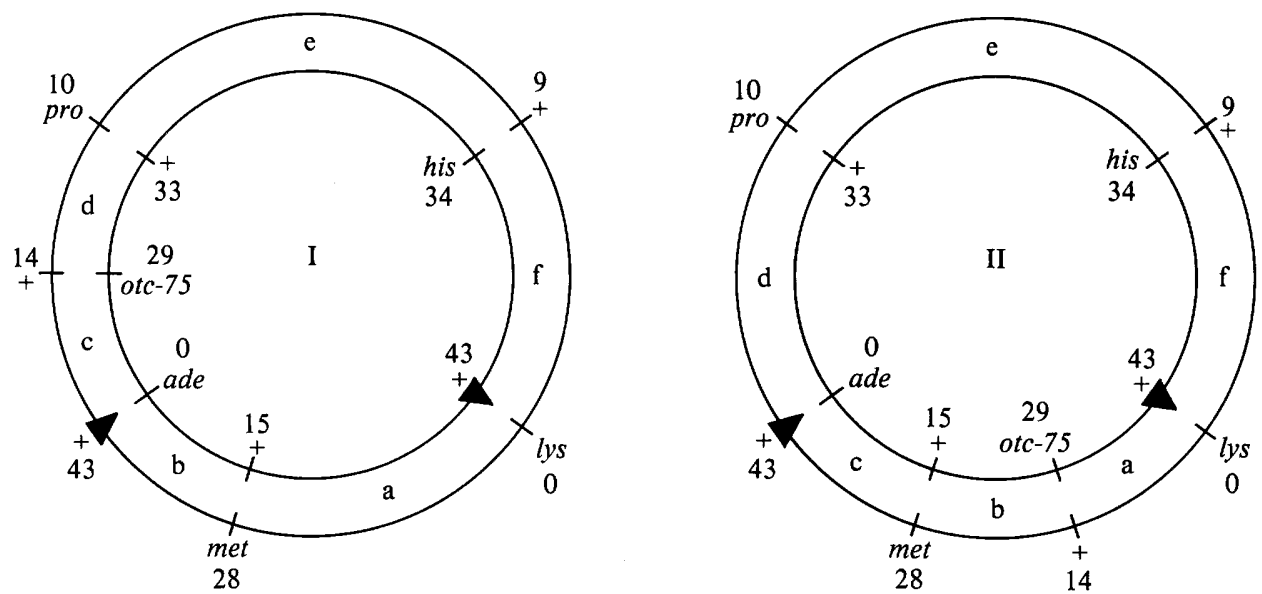

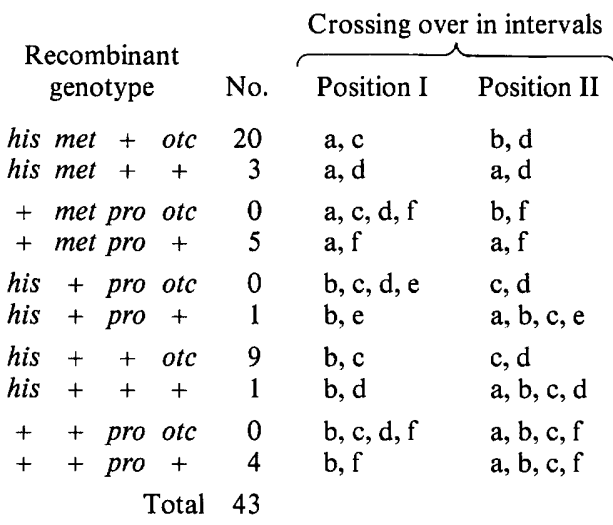

Position $I$ is chosen as the map location of otc-75 since it requires only two crossovers to generate all the observed recombinants, while position II would require four crossovers to generate six progeny belonging to three recombinant classes.

\section{Genetic mapping}

The position of otc and $c s f$ loci on the linkage map of $S$. rimosus was investigated by haploid recombinant analysis (Hopwood, 1967 b). In preliminary experiments, an OTC non-producing mutant was crossed with an OTC producing strain carrying complementary genetic markers. In this way mutants in otcD4, otc $X 20$, otc $Y 90$ and otcZ151 were positioned between $\arg A 1$ and $l y s B 2$, and $\operatorname{csf} A 19, \operatorname{csfB} 56$ and $\operatorname{csfC} 155$ were located between hisA1 and adeA2 (Fig. 2). A further series of crosses involving markers within these two broad regions of the chromosome enabled the same two groups of mutations to be allocated to shorter regions, namely $r i b B 1$ to $c y s D 3$ and proA1 to adeA2 (Fig. 2). The results for two of these crosses, which enabled the locations of otcD4 and otcC75 to be determined, are given in Tables 4 and 5, respectively.

The type of genetic analysis employed does not provide map distances, but the tendency for clustering of the genes involved in OTC and CSF1 biosynthesis was clearly demonstrated. No evidence for plasmid involvement in OTC production was obtained since every otc mutation studied showed clear evidence of chromosomal linkage. 


\section{DISCUSSION}

From our collection of 57 OTC non-producing mutants, it has been possible to select individuals impaired in one of six different steps in the biosynthetic sequence leading to this antibiotic. The mutations are thought to be located in the structural genes of the enzymes responsible for these steps, since all the mutants were involved in specific cosynthetic reactions with other mutants, but definitive proof of this assumption would require the isolation and purification of the enzymes. The nature of the impaired step was defined in three mutants by determining their ability to convert known precursors into OTC. In this way, all the expected types of point-blocked mutants after 4-amino-ATC were found. No mutants which were unable to hydroxylate DHTC in the 5-position were found, but these would not have had the antibiotic-negative phenotype sought in the mutant screening procedure, since they would have produced the antibiotic tetracycline. Three other mutants, all blocked prior to 4-amino-ATC were shown to be blocked in different steps by their ability to cosynthesize with each other. Feeding experiments with early precursors failed to demonstrate conversion, and the blocked steps could not be determined, perhaps because the compounds failed to reach the required intracellular site.

Strong evidence has been presented that a number of mutants impaired in the final step in OTC biosynthesis lacked an essential cofactor (CSF1) for this reaction. At least three different blocks in CSF 1 biosynthesis were demonstrated in mixed culture cosynthesis tests, or using the agar strip test of Delic et al. (1969). These workers, however, concluded that class 1 mutants were altered in 'structural genes involved in the main pathway of antibiotic synthesis'. It therefore seems possible that the OTC non-producing mutants subsequently studied by this group (Alačević, 1976; Pigac et al., 1974) were in fact CSF1-deficient mutants. Conversely, class 2 mutants, which Delic et al. (1969) suggested might be altered in genes not directly involved in the main pathway of antibiotic synthesis, have been shown to be impaired at several different points in that pathway.

Genetic mapping of otc and $c s f$ genes has revealed two chromosomal locations, in agreement with the early work of Boronin \& Mindlin (1971), who were, however, unable to locate their two regions on a general linkage map. Only a single location was reported by Pigac et al. (1974) for seven genes in a chromosomal region partially overlapping one of those determined here (Fig. 2), a result consistent with the hypothesis that these may in fact have been CSF 1 genes. In the present study, mutations in the OTC pathway after ATC, and all CSF 1 mutations, mapped in a single region of the chromosome between proA3 and adeA2. Mutations before ATC mapped to a second region, diametrically opposite the first on the circular chromosome, between cysD3 and ribB1. Clustering and $180^{\circ}$ symmetry of biosynthetically related genes of primary metabolism has been observed in several Streptomyces species (Hopwood, 1967a; Friend \& Hopwood, 1971) but only limited information is available on the location of antibiotic genes (Hopwood \& Merrick, 1977). A single gene cluster was found for actinorhodin genes in $S$. coelicolor (Rudd \& Hopwood, 1979). In the same organism the genes for methylenomycin A biosynthesis were located on a plasmid, SCP1 (Kirby \& Hopwood, 1977).

The symmetry of gene clusters in Streptomyces may be a reflection of an evolutionary increase in chromosome size by duplication (Hopwood, 1967 b). If this theory is correct, the foundations of the OTC pathway must have been laid prior to this event. If the OTC pathway has evolved in a similar manner to primary pathways, this seems to imply a function for OTC in the evolutionary survival of the $S$. rimosus species. This conclusion is also consistent with the fact that a fully antibiotically active compound is only formed in the final step of the complex OTC pathway.

It is hoped that basic studies in biochemical genetics of antibiotic synthesis will lead to an improved understanding of the regulation of these pathways, and of the evolutionary and functional significance of these so-called secondary metabolites. Such studies should also assist the application of recent techniques for genetic manipulation in Streptomyces, such as 
co-mutation (Randazzo et al., 1973), protoplast fusion (Hopwood \& Wright, 1978) and gene cloning via transformation by plasmid DNA (Bibb et al., 1978), to the construction of high-yielding industrial strains and strains producing novel compounds. Work along these lines is in progress in our laboratories.

We would like to thank Mr D. Baldock, Dr D. E. F. Harrison, Professor D. A. Hopwood and Professor R. Thomas for many useful discussions, and for their critical reading of the manuscript. We are also grateful to $\mathrm{Mr} \mathrm{G}$. Clements, Miss T. Frampton, Mr J. Tiley and Miss W. Tolley for skilled technical assistance.

\section{REFERENCES}

AlAČEvić, M. (1976). Recent advances in Streptomyces rimosus genetics. In Second International Symposium on the Genetics of Industrial Microorganisms, pp. 513-519. Edited by K. D. Macdonald. London: Academic Press.

Alač́ević, M., Strašek-Vešlligaj, M. \& Sermonti, G. (1973). The circular linkage map of Streptomyces rimosus. Journal of General Microbiology 77, 173-185.

Alikhanian, S. I., Orlova, N. V., Mindlin, S. Z. \& Zertseva, Z. M. (1961). Genetic control of oxytetracycline biosynthesis. Nature, London 189, 939-940.

BibB, M. J., WARD, J. M. \& Hopwood, D. A. (1978). Transformation of plasmid DNA into Streptomyces at high frequency. Nature, London 274, 398-400.

Blumauerova, M., HostaleK, Z. \& VANEK, Z. (1972). Biosynthesis of tetracyclines: problems and perspectives in genetic analysis. In Fermentation Technology Today, pp. 223-232. Edited by G. Terui. Osaka: Osaka Society of Fermentation Technology.

Boronin, A. M. \& Mindlin, S. Z. (1971). Genetic analysis of Actinomyces rimosus mutants with impaired synthesis of antibiotic. Genetika 8, 125131 (in Russian).

Delic, V., Pigac, J. \& Sermonti, G. (1969). Detection and study of cosynthesis of tetracycline antibiotics by an agar method. Journal of General Microbiology 55, 103-108.

Delić, V., Hopwood, D. A. \& Friend, E. J. (1970). Mutagenesis by $N$-methyl- $N^{\prime}$-nitro- $N$-nitrosoguanidine in Streptomyces coelicolor. Mutation Research 9, 167-182.

FrIEND, E. J. \& Hopwood, D. A. (1971). The linkage map of Streptomyces rimosus. Journal of General Microbiology 68, 187-197.

FrIEND, E. J., WARREN, M. \& Hopwood, D. A. (1978). Genetic evidence for a plasmid controlling fertility in an industrial strain of Streptomyces rimosus. Journal of General Microbiology 106, 201-206.

HopwOOD, D. A. (1967a). In discussion to F. W. Stahl's paper: Circular genetic maps. Journal of Cellular Physiology 70, Suppl. 1, 1-12.

HopwOoD, D. A. $(1967 b)$. Genetic analysis and genome structure in Streptomyces coelicolor. Bacteriological Reviews 31, 373-403.

HopwOOD, D. A. \& MerRICK, M. J. (1977). Genetics of antibiotic production. Bacteriological Reviews 41, 595-635.

HoPWOOD, D. A. \& WRIGHT, H. M. (1978). Bacterial protoplast fusion: recombination in fused proto- plasts of Streptomyces coelicolor. Molecular and General Genetics 162, 307-317.

KIRBY, R. \& HOPWOOD, D. A. (1977). Genetic determination of methylenomycin synthesis by the SCP1 plasmid of Streptomyces coelicolor A3(2). Journal of General Microbiology 98, 239-252.

Martin, J. H., Mitscher, L. A., Miller, P. A., Shu, P. \& Bohonos, N. (1966). 5-Hydroxy-7-chlorotetracycline. I. Preparation, isolation and physicochemical properties. Antimicrobial Agents and Chemotherapy, 563-567.

McCormick, J. R. D. (1966). Biosynthesis of the tetracyclines: an integrated biosynthetic scheme. In Antibiotics-Advances in Research, Production and Clinical Use, pp. 556-573. Edited by M. Herold \& Z. Gabriel. Prague: Czechoslovak Medical Press.

McCoRmiCK, J. R. D. (1968). Point blocked mutants and the biogenesis of tetracyclines. In Genetics and Breeding of Streptomyces, pp. 163-176. Edited by 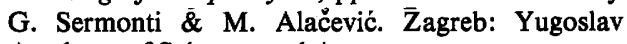 Academy of Sciences and Arts.

MCCORMICK, J. R. D., HirsCh, U., SJOlander, N. O. \& Doerschuk, A. P. (1960). Cosynthesis of tetracyclines by pairs of Streptomyces aureofaciens mutants. Journal of the American Chemical Society 82, 5006-5007.

Miller, P. A., SJolander, N. O., NalesynK, S., ARnold, N., Johnson, S., Doerschuk, A. P. \& MCCoRmick, J. R. D. (1960). Cosynthetic factor 1, a factor in hydrogen-transfer in Streptomyces aureofaciens. Journal of the American Chemical Society 82, 5002-5003.

MilleR, P. A., HASh, J. H., Lincks, M. \& Bohonos, N. (1965). Biosynthesis of 5-hydroxytetracycline. Biochemical and Biophysical Research Communications 18, 325-331.

Pigac, J., VešligaJ, M. \& Delić, V. (1974). Mapping of oxytetracycline genes in Streptomyces rimosus. In Genetics of Industrial Microorganisms, Abstracts of the Second International Symposium, p. 105. London: Academic Press.

Randazzo, R., Sermonti, G., Carere, A. \& Bignam, M. (1973). Comutation in Streptomyces. Journal of Bacteriology 113, 500-501.

RuDD, B. A. M. \& Hopwood, D. A. (1979). Genetics of actinorhodin biosynthesis by Streptomyces coelicolor A3(2). Journal of General Microbiology 114, 35-43.

Townsend, M. E., Wright, H. M. \& Hopwood, D. A. (1971). Efficient mutagenesis by near ultraviolet light in the presence of 8-methoxypsoralen in Streptomyces. Journal of Applied Bacteriology 34, 799-801. 